\title{
SENSE: Sensitivity Encoding for Fast MRI
}

\author{
Klaas P. Pruessmann, Markus Weiger, Markus B. Scheidegger, and Peter Boesiger*
}

\begin{abstract}
New theoretical and practical concepts are presented for considerably enhancing the performance of magnetic resonance imaging (MRI) by means of arrays of multiple receiver coils. Sensitivity encoding (SENSE) is based on the fact that receiver sensitivity generally has an encoding effect complementary to Fourier preparation by linear field gradients. Thus, by using multiple receiver coils in parallel scan time in Fourier imaging can be considerably reduced. The problem of image reconstruction from sensitivity encoded data is formulated in a general fashion and solved for arbitrary coil configurations and $k$-space sampling patterns. Special attention is given to the currently most practical case, namely, sampling a common Cartesian grid with reduced density. For this case the feasibility of the proposed methods was verified both in vitro and in vivo. Scan time was reduced to one-half using a two-coil array in brain imaging. With an array of five coils double-oblique heart images were obtained in one-third of conventional scan time. Magn Reson Med 42:952-962, 1999. ㅅ 1999 Wiley-Liss, Inc.
\end{abstract}

Key words: MRI; sensitivity encoding; SENSE; fast imaging; receiver coil array

Among today's many medical imaging techniques, MRI stands out by a rarely stated peculiarity: the size of the details resolved with MRI is much smaller than the wavelength of the radiation involved. The reason for this surprising ability is that the origin of a resonance signal is not determined by optical means such as focusing or collimation but by spectral analysis. The idea of Lauterbur (1) to encode object contrast in the resonance spectrum by a magnetic field gradient forms the exclusive basis of signal localization in Fourier imaging. However powerful, the gradient-encoding concept implies a fundamental restriction. Only one position in $k$-space can be sampled at a time, making $k$-space speed the crucial determinant of scan time. Accordingly, gradient performance has been greatly enhanced in the past, reducing minimum scan time drastically with respect to earlier stages of the technique. However, due to both physiological and technical concerns, inherent limits of $k$-space speed have almost been reached.

An entirely different approach to sub-wavelength resolution in MRI is based on the fact that with a receiver placed near the object the contribution of a signal source to the induced voltage varies appreciably with its relative position. That is, knowledge of spatial receiver sensitivity implies information about the origin of detected MR signals, which may be utilized for image generation. Unlike position in $k$-space, sensitivity is a receiver property and does not refer to the state of the object under examination.

Institute of Biomedical Engineering and Medical Informatics, University of Zürich and Swiss Federal Institute of Technology Zürich, Zürich, Switzerland.

Grant sponsor: EUREKA; Grant number: EU1353; Grant sponsor: KTI; Grant number: 3030.2 .

${ }^{*}$ Correspondence to: Prof. Dr. P. Boesiger, Institute of Biomedical Engineering and Medical Informatics, University and ETH Zürich, Gloriastrasse 35, CH-8092 Zürich Switzerland.

Received 30 July 1998; revised 18 May 1999; accepted 9 July 1999.

(c) 1999 Wiley-Liss, Inc.
Therefore, samples of distinct information content can be obtained at one time by using distinct receivers in parallel (2), implying the possibility of reducing scan time in Fourier imaging without having to travel faster in $k$-space.

In 1988 Hutchinson and Raff (3) suggested dispensing entirely with phase encoding steps in Fourier imaging by using a very large number of receivers. Kwiat et al. (4) proposed a similar concept in 1991. In 1989 Kelton et al. (5) suggested staying with phase encoding, yet reducing the number of phase encoding steps by a power of 2 using a corresponding number of receivers. In Kelton et al. (5), as in all later concepts, phase encoding is reduced by increasing the distance of readout lines in $k$-space such that the sampled area remains unchanged. The Kelton approach was modified by Ra et al. (6) in 1991, allowing the number of coils to be any integer, yet still equal to the factor of scan time reduction.

In all contributions procedures for image reconstruction were derived. However, applications of the concepts noted have not been reported, reflecting the considerable practical challenges of sensitivity based imaging, including the signal-to-noise ratio (SNR) issue, sensitivity assessment, and hardware requirements. Only in 1997 did Sodickson et al. (7) report the first successful experiments using parallel receivers for the purpose of scan time reduction, introducing the SMASH method (SiMultaneous Acquisition of Spatial Harmonics). For image reconstruction SMASH relies on the ability to approximate low-order harmonics of the desired field of view (FOV) by linear combination of sensitivity functions. The technique is therefore restricted to appropriate combinations of coil arrangement, slice geometry, and reduction factor.

To overcome the restrictions of previously proposed methods, in this work we reformulate the problem of image reconstruction from multiple receiver data. Using the framework of linear algebra, two different reconstruction strategies have been derived. In their general forms the resulting formulae hold for arbitrary sampling patterns in $k$-space. A detailed discussion is dedicated to the most practical case, namely, sampling along a Cartesian grid in $k$-space corresponding to standard Fourier imaging with reduced FOV.

Owing to the underlying principle, the concepts outlined in this work have been named SENSE, short for SENSitivity Encoding (8-10). Together with SENSE theory and methods, a detailed SNR analysis is presented as well as an experimental in vitro evaluation and a selection of in vivo examples.

\section{THEORY AND METHODS}

In this section SENSE theory is presented and methods for image reconstruction from sensitivity encoded data are derived. The theory addresses the most general case of combining gradient and sensitivity encoding. That is, no 
restrictions are made as to the coil configuration and the sampling pattern in $k$-space. Two reconstruction strategies are discussed. The first approach strictly aims at optimal voxel shape and is called strong reconstruction for convenience. In weak reconstruction, the voxel shape criterion is weaker in favor of the SNR. With both strategies the reconstruction algorithm is numerically demanding in the general case. This is mainly because with hybrid encoding the bulk of the work of reconstruction can usually not be done by fast Fourier transform (FFT).

However, it is shown that in weak reconstruction FFT can still be applied if $k$-space is sampled in a regular Cartesian fashion. For this reason sensitivity encoding with Cartesian sampling is particularly feasible. Moreover, the reconstruction mechanism is relatively easily understood in this case. Therefore, the first part of this section gives a practical description of the Cartesian case. The following parts are dedicated to general theory, SNR and error considerations, and sensitivity assessment.

\section{Sensitivity Encoding With Cartesian Sampling of $k$-Space}

In two-dimensional (2D) Fourier imaging with common Cartesian sampling of $k$-space, sensitivity encoding by means of a receiver array permits reduction of the number of Fourier encoding steps. This is achieved by increasing the distance of sampling positions in $k$-space while maintaining the maximum $k$-values. Thus scan time is reduced at preserved spatial resolution. The factor by which the number of $k$-space samples is reduced is referred to as the reduction factor $R$. In standard Fourier imaging, reducing the sampling density results in the reduction of the FOV, causing aliasing. In fact, SENSE reconstruction in the Cartesian case is efficiently performed by first creating one such aliased image for each array element using discrete Fourier transform (DFT).

The second step then is to create a full-FOV image from the set of intermediate images. To achieve this one must undo the signal superposition underlying the fold-over effect. That is, for each pixel in the reduced FOV the signal contributions from a number of positions in the full FOV need to be separated. As depicted in Fig. 1, these positions form a Cartesian grid corresponding to the size of the reduced FOV.

The key to signal separation lies in the fact that in each single-coil image signal superposition occurs with different weights according to local coil sensitivities. Consider one pixel in the reduced FOV and the corresponding set of pixels in the full FOV (Fig. 1). Let $n_{\mathrm{P}}$ denote the number of pixels superimposed and $n_{\mathrm{C}}$ the number of coils used. Assemble in the vector $\boldsymbol{a}$ the complex image values the chosen pixel has in the intermediate images. The complex coil sensitivities at the $n_{\mathrm{P}}$ superimposed positions form an $n_{\mathrm{C}} \times n_{\mathrm{P}}$ sensitivity matrix $S$ :

$$
S_{\gamma, \rho}=s_{\gamma}\left(\boldsymbol{r}_{\rho}\right),
$$

where the subscripts $\gamma, \rho$ count the coils and the superimposed pixels, respectively, $\boldsymbol{r}_{\rho}$ denotes the position of the pixel $\rho$, and $s_{\gamma}$ is the spatial sensitivity of the coil $\gamma$. The sensitivity matrix is used to calculate the unfolding ma-

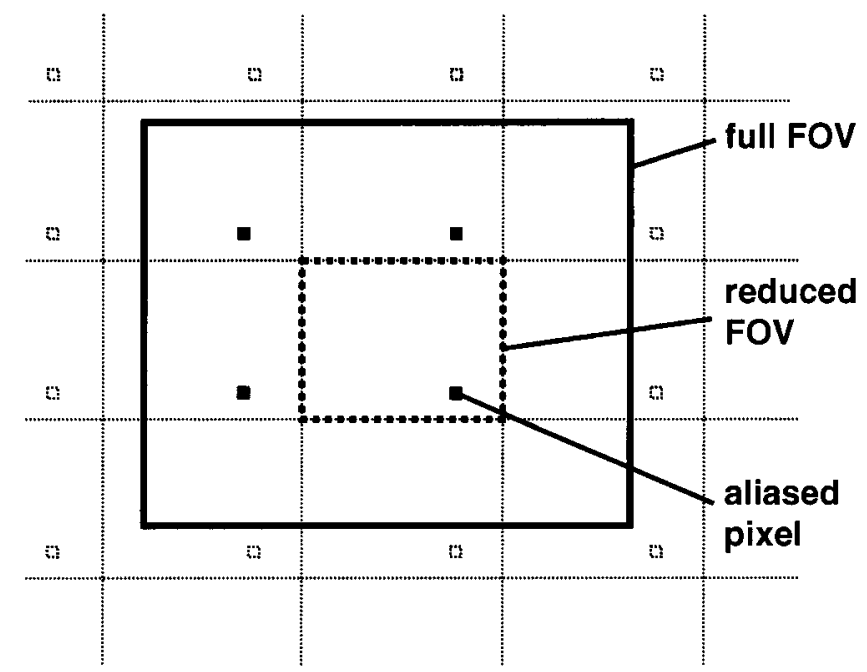

FIG. 1. Aliasing in 2D Cartesian sampling: the full FOV (solid box) is reduced in both dimensions. A pixel in the reduced FOV (dotted box) represents the superposition of pixels forming a Cartesian grid. In this example four of these pixels are in the full FOV; thus the actual degree of aliasing is four.

$\operatorname{trix} U$ :

$$
U=\left(S^{H} \Psi^{-1} S\right)^{-1} S^{H} \Psi^{-1},
$$

where the superscript $H$ indicates the transposed complex conjugate, and $\Psi$ is the $n_{\mathrm{C}} \times n_{\mathrm{C}}$ receiver noise matrix (see Appendix A), which describes the levels and correlation of noise in the receiver channels. Using the unfolding matrix, signal separation is performed by

$$
\boldsymbol{v}=U \boldsymbol{a},
$$

where the resulting vector $\mathbf{v}$ has length $n_{\mathrm{P}}$ and lists separated pixel values for the originally superimposed positions. By repeating this procedure for each pixel in the reduced FOV a non-aliased full-FOV image is obtained.

Unfolding is possible as long as the inversions in Eq. [2] can be performed. In particular, the number of pixels to be separated, $n_{\mathrm{P}}$, must not exceed $n_{\mathrm{C}}$. In other words, the reduction factor is bound by the number of coils used. Note that $n_{\mathrm{P}}$ does not need to be the same for all partial unfolding steps. Upon non-integer reduction the number of pixels actually superimposed may vary in the reduced FOV. Generally, the degree of aliasing plays an important role with respect to SNR. As a rule of thumb it can be said that local SNR improves when the degree of aliasing is reduced. Therefore it is beneficial to exclude a pixel from reconstruction when the corresponding volume contributes no signal, e.g., because it lies outside of the object. Formally this is done simply by removing the corresponding column in the sensitivity matrix and setting the excluded pixel to zero in the final image. Knowledge of which voxels may safely be excluded is obtained as a by-product of sensitivity assessment.

In Eq. [2] receiver noise levels and correlation are considered for the sake of SNR optimization. Optionally, the assessment of receiver noise may be skipped and the 
matrix $\Psi$ replaced by identity. Then unfolding is still ensured, yet at an SNR penalty, which generally will be the more marked the less equivalent the receivers are with respect to load, gain, and mutual coupling. Equation [2] holds independently of whether foldover occurs in one or two directions. Clearly, for the sake of scan time reduction in 2D imaging, undersampling is reasonable only in the phase encoding direction. However, in techniques using two phase encoding directions the independent variation of two reduction factors adds a valuable degree of freedom.

\section{General Theory of Sensitivity Encoding}

Consider an imaging experiment using an array of $n_{\mathrm{C}}$ receiver coils. Fourier encoding is described by a set of $n_{K}$ sampling positions in $k$-space. Let the whole object be within the volume of interest (VOI). Then a sample value $m$ obtained from the $\gamma$-th coil at the $\mathrm{\kappa}$-th position in $k$-space is given by

$$
m_{\gamma, \mathrm{\kappa}}=\int_{\mathrm{VOI}} c(\boldsymbol{r}) e_{\gamma, \mathrm{\kappa}}(\boldsymbol{r}) d \boldsymbol{r}
$$

where $\boldsymbol{r}$ denotes 3D position,

$$
e_{\gamma, \kappa}(\boldsymbol{r})=e^{i \boldsymbol{k}_{\mathrm{k}} \boldsymbol{r}} S_{\gamma}(\boldsymbol{r})
$$

is the net encoding function composed of harmonic modulation and the complex spatial sensitivity $s_{\gamma}$ of coil $\gamma$, and $c$ results from tissue and sequence parameters. The effects of non-uniform $k$-space weighting due to relaxation shall be neglected in the scope of this work.

From the linearity of encoding it is clear that image reconstruction must essentially be linear as well. That is, each of $n_{\mathrm{V}}$ image values is to be calculated as a linear combination of sample values:

$$
V_{\rho}=\sum_{\gamma, \kappa} F_{\rho,(\gamma, \kappa)} m_{\gamma, \kappa}
$$

where $\rho$ counts the voxels to be resolved. The transform $F$ shall be referred to as the reconstruction matrix. Its size is $n_{\mathrm{V}} \times n_{\mathrm{C}} n_{\mathrm{K}}$. Assembling sample and image values in vectors, image reconstruction may be rewritten in matrix notation:

$$
\boldsymbol{v}=F \boldsymbol{m} .
$$

With such linear mapping the propagation of noise from sample values into image values is conveniently described by noise matrices. The $\rho$-th diagonal entry of the image noise matrix $X$ represents the noise variance in the $\rho$-th image value while the off-diagonal entries reflect noise correlation between image values. The sample noise matrix $\tilde{\Psi}$ is defined accordingly in Appendix A. As shown there, these matrices fulfil the relation

$$
X=F \tilde{\Psi} F^{H}
$$

The central objective in choosing a reconstruction matrix is to make each image value selectively reflect signal from the voxel it represents. To trace the origin of signal in image values, insert Eq. [4] into Eq. [6], to find

$$
v_{\rho}=\int_{\text {VOI }} C(\boldsymbol{r})\left(\sum_{\gamma, \mathrm{\kappa}} F_{\rho,(\gamma, \mathrm{\kappa})} e_{\gamma, \mathrm{\kappa}}(\boldsymbol{r})\right) d \boldsymbol{r} .
$$

The term in brackets describes the spatial weighting of signal in $v_{\rho}$. It is therefore called the corresponding voxel function:

$$
f_{\rho}(\boldsymbol{r})=\sum_{\gamma, \kappa} F_{\rho,(\gamma, \kappa)} e_{\gamma, \kappa}(\boldsymbol{r})
$$

Hence, the matrix $F$ has to be chosen such that the resulting voxel functions approximate the desired voxel shapes. Let $i_{\rho}(\boldsymbol{r})$ denote an orthonormal set of ideal voxel shapes, e.g., box functions. The relation between ideal voxel shapes and encoding functions is described by the $n_{\mathrm{C}} n_{\mathrm{K}} \times n_{\mathrm{V}}$ encoding matrix

$$
E_{(\gamma, \kappa), \rho}=\int_{\text {VOI }} i_{\rho}^{*}(\boldsymbol{r}) e_{\gamma, \kappa}(\boldsymbol{r}) d \boldsymbol{r} .
$$

There are many possible ways of approximating ideal voxels. Here we discuss two concepts. The first approach is to choose those voxel functions that exhibit the least square deviation from the ideal. This criterion entirely determines reconstruction; the approach is therefore referred to as the strong one. In Appendix B it is shown that it yields

$$
F=E^{H} C^{-1},
$$

where $C$ denotes the correlation matrix of the encoding functions. The image noise matrix [8] is then given by

$$
X=E^{H} C^{-1} \tilde{\Psi} C^{-1} E
$$

The second approach, dubbed the weak one, uses a different concept of similarity between real voxel functions and ideal shapes. It requires that each voxel function fulfil the orthonormality relations of its ideal counterpart:

$$
\int_{\text {VOI }} i_{\rho}^{*}(\boldsymbol{r}) f_{\rho^{\prime}}(\boldsymbol{r}) d \boldsymbol{r}=\delta_{\rho, \rho^{\prime}} \quad \forall \rho, \rho^{\prime} .
$$

Using Eqs. [10] and [11], Eq. [14] may be rewritten in matrix form:

$$
F E=I d_{n_{v}}
$$

where $I d_{n_{\mathrm{V}}}$ denotes $n_{\mathrm{V}} \times n_{\mathrm{V}}$ identity. By this condition the reconstruction matrix $F$ is generally not yet entirely determined. It leaves $n_{\mathrm{C}} n_{\mathrm{K}}-n_{\mathrm{V}}$ degrees of freedom per voxel, which may be utilized for SNR optimization. To that end each diagonal element of the image noise matrix $X$ is minimized under condition [15] by Lagrange calculus (see Appendix C), yielding

$$
F=\left(E^{H} \tilde{\Psi}^{-1} E\right)^{-1} E^{H} \tilde{\Psi}^{-1}
$$


In this case the image noise matrix reads

$$
X=\left(E^{H} \tilde{\Psi}^{-1} E\right)^{-1}
$$

The reconstruction formulae [12] and [16] permit image reconstruction from data obtained with hybrid gradient and sensitivity encoding. Both are numerically challenging as they imply the inversion of large matrices. However, the two concepts also exhibit important differences. The weak approach is more convenient in that it does not require the calculation of the matrix $C$ and poses the smaller inversion problem when $n_{\mathrm{V}}<n_{\mathrm{C}} n_{\mathrm{K}}$. Furthermore, it yields optimized SNR. On the other hand, the strong approach is always applicable, whereas the second algorithm works only if condition [15] can be fulfilled. In particular, for weak reconstruction the rank of the matrix $E$ must be equal to $n_{\mathrm{V}}$, thus $n_{\mathrm{V}} \leqslant n_{\mathrm{C}} n_{\mathrm{K}}$ must hold. Moreover, the weak approach is less robust in terms of ensuring voxel quality.

The limitations of weak reconstruction may be understood by considering Dirac distributions as ideal voxel functions:

$$
i_{\rho}(\boldsymbol{r})=\delta\left(\boldsymbol{r}-\boldsymbol{r}_{\rho}\right),
$$

where $\boldsymbol{r}_{\rho}$ denotes the center of the $\rho$-th voxel. The encoding matrix then reduces to

$$
E_{(\gamma, \mathrm{\kappa}), \rho}=e_{\gamma, \kappa}\left(\boldsymbol{r}_{\rho}\right)
$$

In this case the weak criterion [15] may be restated as follows: each voxel function must be equal to one in the center of the voxel it belongs to and equal to zero in all other voxels' centers. A voxel function with this property will be acceptable only as long as it is well behaved between voxel centers. In this view, the criterion becomes unreliable when there are solutions that vary considerably within voxels and at the same time yield favorably low noise.

The Dirac choice in the weak approach also is of great practical significance. It is with this choice that reconstruction in the Cartesian case can be performed in the practical fashion described at the beginning of this section. For the derivation see Appendix D.

\section{Noise in SENSE Images}

There are actually two kinds of noise that affect SENSE images, i.e., noise in sample values and noise in sensitivity data. The latter, however, can usually be reduced to a negligible level by smoothing. Then Eq. [8] for the calculation of image noise holds. This equation illustrates two important aspects of noise propagation in SENSE reconstruction. First, with multiple channels the diagonal entries in $\tilde{\Psi}$ vary from channel to channel and there is noise correlation between samples taken simultaneously, i.e., there are non-zero cross-terms. Second, unlike a matrix representation of FFT, a SENSE reconstruction matrix generally is not unitary. As a consequence, unlike standard Fourier images the noise level in a SENSE image varies from pixel to pixel and there is noise correlation between pixels.

For similar reasons the noise level does not have the common square-root dependence on the number of samples taken. In the case of Cartesian sampling with reconstruction as initially explained, this can be made yet clearer. For one particular voxel we compare the noise levels as obtained with full and reduced Cartesian Fourier encoding. According to Appendix D the partial image noise matrix for the relevant unfolding step is

$$
X=\frac{1}{n_{K}}\left(S^{H} \Psi^{-1} S\right)^{-1}
$$

Let $\rho$ denote the index of the voxel under consideration within the set of voxels to be separated. With full Fourier encoding no aliasing occurs and the matrix $S$ has only one column. Note that this single column is identical to the $\rho$-th column of $S$ in the case of reduced Fourier encoding. Thus, the ratio of the noise levels obtained in that voxel is given by

$$
\frac{\sqrt{X_{\rho, \rho}^{\text {red }}}}{\sqrt{X_{\rho, \rho}^{\text {full }}}}=\sqrt{R} \sqrt{\left[\left(S^{H} \Psi^{-1} S\right)^{-1}\right]_{\rho, \rho}\left(S^{H} \Psi^{-1} S\right)_{\rho, \rho}},
$$

where $S$ corresponds to reduced Fourier encoding and $R$ denotes the factor by which the number of samples is reduced with respect to full Fourier encoding:

$$
R=\frac{n_{K}^{f u l l}}{n_{K}^{r e d}}
$$

The rightmost square-root expression in Eq. [21] strongly depends on coil geometry and is thus called the local geometry factor $g$, which is always at least equal to one:

$$
g_{\rho}=\sqrt{\left[\left(S^{H} \Psi^{-1} S\right)^{-1}\right]_{\rho, \rho}\left(S^{H} \Psi^{-1} S\right)_{\rho, \rho}} \geq 1 .
$$

Note that by virtue of condition [15] the voxel functions in the two reconstructions compared are both scaled to one in the voxel center. Therefore, the noise ratio in Eq. [21] reflects just the inverse of the SNR ratio, thus:

$$
S N R_{\rho}^{\text {red }}=\frac{S N R_{\rho}^{\text {full }}}{g_{\rho} \sqrt{R}} .
$$

This relation confirms an upper bound for SNR characterized by the square root of the number of samples acquired. The geometry factor describes the ability with the used coil configuration to separate pixels superimposed by aliasing. In practice it allows a priori SNR estimates and provides an important criterion for the design of dedicated coil arrays.

\section{Propagation of Systematic Error in SENSE Reconstruction}

In addition to noise a SENSE reconstructed image may be impaired by errors of systematic nature. Errors in sample values may be due, e.g., to tissue motion, main field inhomogeneity, eddy currents, or gradient non-linearity. The related artifacts are well known in standard imaging. A potential problem specific to sensitivity encoded imaging arises from errors in sensitivity values.

The nature of artifacts in SENSE images generally is governed by error propagation in the reconstruction formu- 
lae. More specific statements are possible in the case of Cartesian sampling with unfolding reconstruction. Here errors in sample values are first reflected in the intermediate images and then undergo mapping by the unfolding matrices $U$. These cause error cross-talk within sets of equidistant voxels, as depicted in Fig. 1. Therefore, the artifacts resulting in the final image basically have the same appearance as in the single coil images, yet occur periodically with varying intensity. In the determination of coil sensitivities the most typical systematic error is regional over- or underestimation. Upon unfolding such deviations give rise to periodical artifacts depicting parts of the object. Generally, the severity of both types of artifacts crucially depends on the condition of $U$.

\section{Determination of Sensitivity Maps}

Sensitivity based reconstruction requires highly accurate sensitivity assessment. To this end concepts known from methods for intensity correction (11-13) have been extended (14). Reliable sensitivity information is obtained by reference measurements with the definitive set-up in addition to actual imaging. With this strategy it is not possible to assess absolute sensitivity values. However, according to Eq. [4], a spatial scaling of sensitivity values, as long as it is the same for all coils, is mapped onto the final image without further interference with reconstruction. Therefore it is sufficient to determine sensitivity maps with equal and appropriately homogeneous scaling.

The first step in generating such maps is to acquire and reconstruct single-coil, full-FOV images of the slice of interest in a conventional manner. Division of each of these images by the "sum-of-squares" of the set yields sensitivity maps with scaling clear of modulus object contrast but still modulated by the "sum-of-squares" of absolute sensitivities. More homogeneous scaling is achieved by dividing by a body coil image. The "sum-of-squares" denominator is applicable only if the object phase is sufficiently smooth so as to endure map refinement largely unaltered. On the other hand, it offers the advantage of being more reliable in clearing modulus object contrast from raw maps. This is because a body coil reference cannot be acquired quite simultaneously with the array reference.

In either case the raw maps obtained are impaired by noise (Fig. 2a-c). Straightforward elimination of noise by low-pass filtering results in errors at object edges (15). To overcome this problem, smoothing is accomplished by a polynomial fit procedure. For each pixel of a raw map a $2 \mathrm{D}$ polynomial is locally fit to the noisy data. A fitting polynomial for the pixel $x_{0}, y_{0}$ is given by

$$
s(x, y)=\sum_{l, m=0}^{P} c_{l, m}\left(x-x_{0}\right)^{l}\left(y-y_{0}\right)^{m},
$$

where $x, y$ are pixel indices, and $P$ denotes the order of the polynomial. Minimizing the weighted square deviation from raw sensitivity values yields a set of $(P+1)^{2}$ equations:

$$
\sum_{l^{\prime}, m^{\prime}=0}^{P} c_{l^{\prime} m^{\prime}} A_{l l^{\prime} m m^{\prime}}=b_{l m},
$$

where

$$
\begin{gathered}
A_{I I^{\prime} m m^{\prime}}=\sum_{x, y} w(x, y)\left(x-x_{0}\right)^{l+l^{\prime}}\left(y-y_{0}\right)^{m+m^{\prime}}, \\
b_{l m}=\sum_{x, y} w(x, y) s_{x, y}\left(x-x_{0}\right)^{l}\left(y-y_{0}\right)^{m},
\end{gathered}
$$

$s_{X, y}$ denotes raw sensitivity values and $w$ is the weighting function. Solving Eq. [26] yields the desired refined sensitivity value for the pixel $x_{0}, y_{0}$. The weighting function reflects the relative significance of $s_{X, y}$ for the refinement of the sensitivity value at $x_{0}, y_{0}$. It is the product of a distance Gaussian and reliability terms derived from analysis of error propagation in creating the raw map:

$$
w(x, y)=e^{-\left[\left(x-x_{0}\right)^{2}+\left(y-y_{0}\right)^{2}\right] / \Omega^{2}}\left|\frac{d_{x, y}}{s_{x, y}}\right| \Phi_{x, y},
$$

where $\Omega$ is a parameter reflecting the degree of smoothing, $d_{x, y}$ denotes the pixel value at position $x, y$ of the denominator image used for preparing the raw map, and $\Phi_{x, y}$ is an "object indicator" map: $\Phi_{x, y}=1$ where object signal dominates noise in the denominator image, $\Phi_{x, y}=0$ elsewhere. $\Phi$ is determined from the denominator image by pixel-wise modulus discrimination with a threshold on the

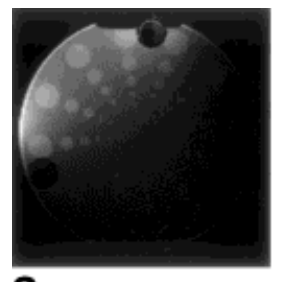

a

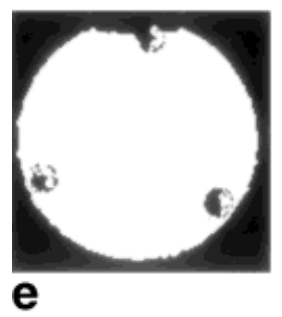

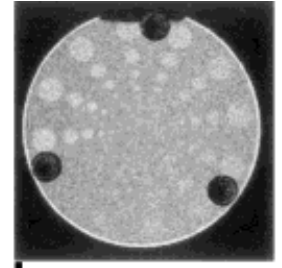

b

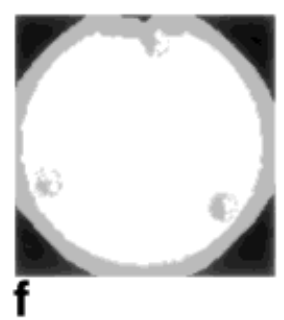

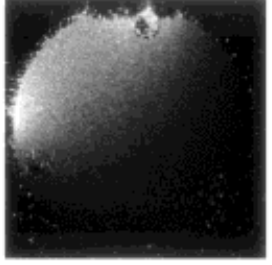

C

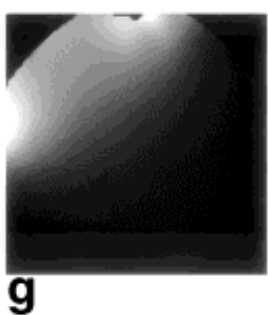

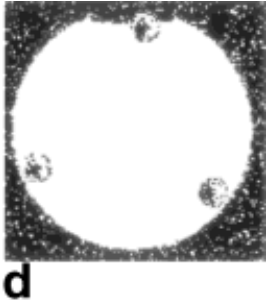

FIG. 2. Determination of sensitivity maps. Division of a surface coil image (a) and a body coil image (b) of the same slice yields a raw sensitivity map (c). Regions exhibiting pure noise are identified by thresholding (d) and subsequent density filtering (e). The extrapolation zone is determined by region growing (f). Local polynomial fitting yields the refined map $(\mathbf{g})$. 
order of the noise level and further exclusion of pixels with then sparse neighborhood (Fig. 2d,e). In addition to the use in Eq. [29] the function $\Phi$ can serve as the basis for excluding voxels from reconstruction.

Besides improved accuracy at object edges, the fitting approach has the advantage of not being restricted to regions yielding immediate sensitivity information. It permits extrapolation over a limited range necessary for dealing with slightly varying tissue configurations. Refined sensitivity values are calculated for all "object" pixels according to $\Phi$, plus an extrapolation zone (Fig. 2f). For efficient implementation note that the sums in Eqs. [27] and [28] are dominated by the pixels near $x_{0}, y_{0}$, the number of considerable contributions depending on $\Omega$. In terms of complexity it is advantageous to consider only the significant terms in Eqs. [27] and [28] and restrict higher order fitting to border regions.

\section{RESULTS}

Sensitivity encoding using common Cartesian sampling of $k$-space and DFT-based reconstruction was performed in vitro and in vivo on a Philips Gyroscan ACS-NT15 at $1.5 \mathrm{~T}$.

\section{Phantom Experiments}

A five-coil array was used in the set-up depicted in Fig. 3a, surrounding a cylindrical quality phantom. A fast gradientfield echo (FFE) sequence was used with a full FOV of 210 $\mathrm{mm}$. SENSE imaging was performed varying the reduction factor from $R=1.0$ (no reduction) up to $R=4.0$ and switching the phase encoding direction between vertical and horizontal. The results are displayed in Figs. 4 and 5, showing conventional "sum-of-squares" and SENSE reconstruction. Throughout, images without appreciable artifacts were obtained, except for noise inhomogeneity visible at higher reduction factors. Maps of the relative noise level as predicted by theory are displayed next to the corresponding images. The maps are in good agreement with visual noise perception and illustrate the benefit of voxel exclusion, as reflected by contours corresponding to object shape. The difference in noise between the two orientations is due to different geometry factors. Obviously, in terms of geometry the vertical phase encoding is the superior choice for the given arrangement of coils and imaged slice.

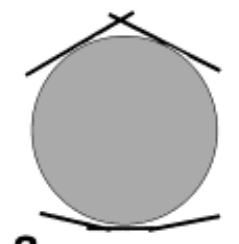

2

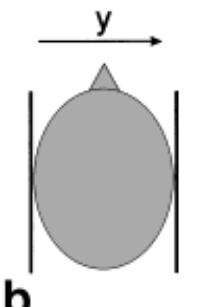

b

FIG. 3. Experimental set-ups. a: A five-coil array positioned around a cylindrical phantom (two overlapping circular elements, $\varnothing 20 \mathrm{~cm}$, three overlapping rectangular elements, $13 \times 19 \mathrm{~cm}$ ). b: Two circular coils $(\varnothing 20 \mathrm{~cm})$ placed laterally for brain imaging. c: Cardiac imaging in prone position with the same array as in a. Arrows indicate the fold-over direction $y$.
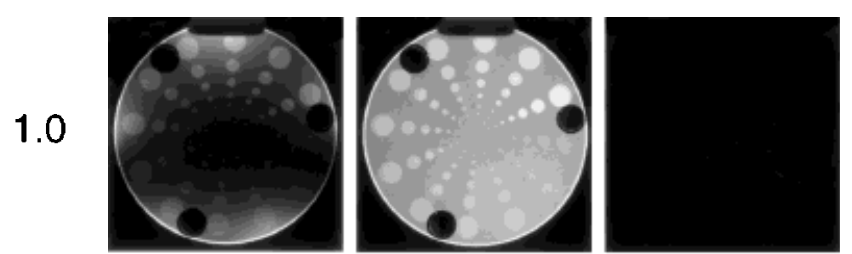

2.0
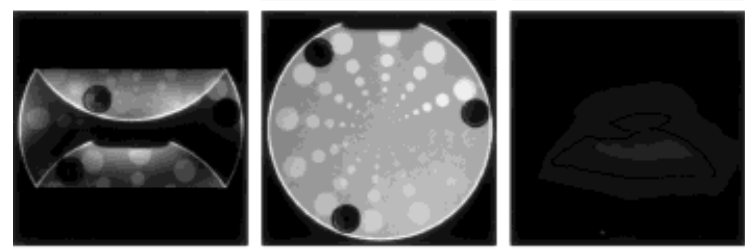

2.4
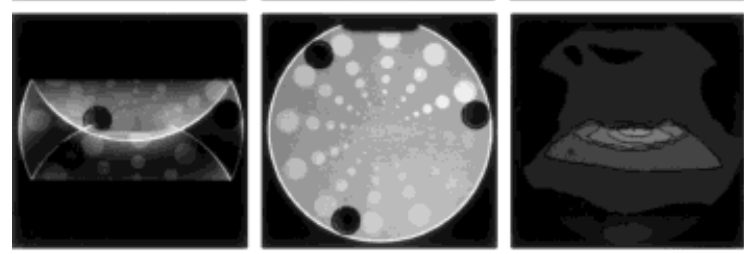

3.0
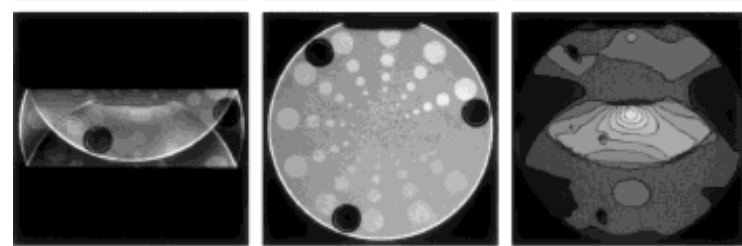

4.0
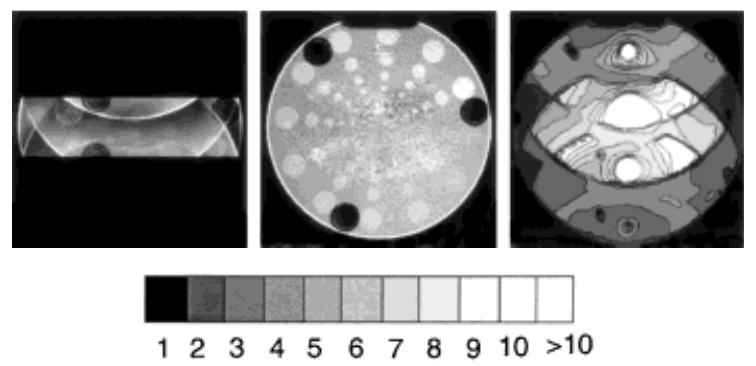

FIG. 4. SENSE imaging of a quality phantom with increasing reduction factor $R$ indicated on the left. Phase encoding in vertical direction. Left: conventional sum-of-squares images. Middle: SENSE reconstruction from the same data. Right: maps of the relative noise level as predicted by SENSE theory, colored according to the gray-scale on the far right (arbitrary units).

To illustrate the need for advanced sensitivity assessment, the fitting order $P$ was varied in sensitivity map refinement. Images obtained at $R=3.0$ with $P=0, P=1$, and $P=2$ are shown in Fig. 6. With zero-order fitting, which is essentially equivalent to low-pass filtering, severe displacement artifacts are observed. In the image obtained with first-order fitting, slight artifacts are still appreciable. By second-order fitting, artifacts were reduced to a negligible level.

\section{In Vivo Experiments}

The elements of a two-coil array were placed laterally on opposite sides of a healthy volunteer's head as depicted in Fig. 3b. Transverse brain images were obtained with full Fourier encoding $(R=1.0)$ and with scan time reduced to one-half $(R=2.0)$ using an inversion recovery turbo spin-echo (IR-TSE) sequence (matrix $256 \times 208$, FOV 210 $\mathrm{mm}$, TI $150 \mathrm{msec}$, TE $11 \mathrm{msec}$, TR $3.5 \mathrm{sec}$, flip angle 90, 17 echoes per echo train, slice thickness $5 \mathrm{~mm}$, NSA 4). 
1.0
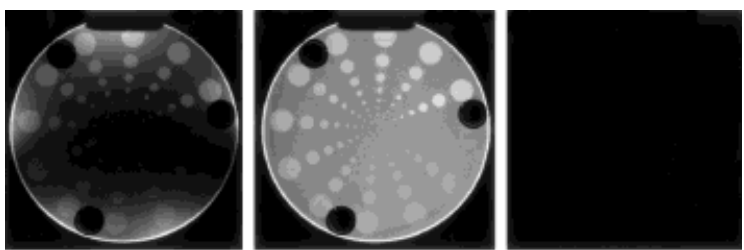

2.0
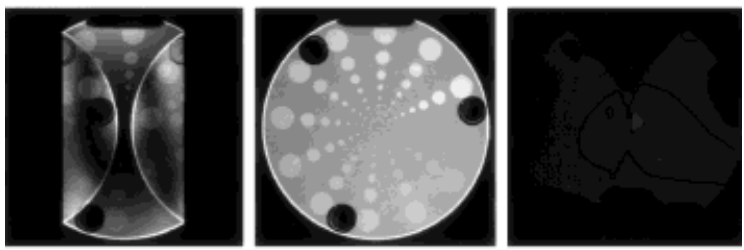

2.4
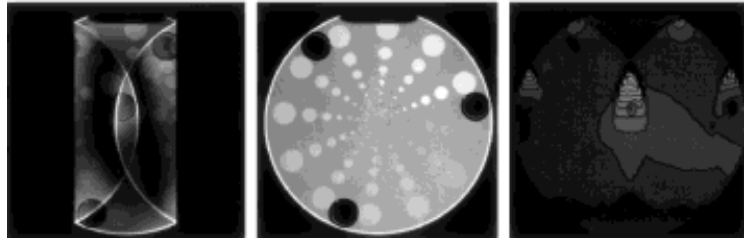

3.0
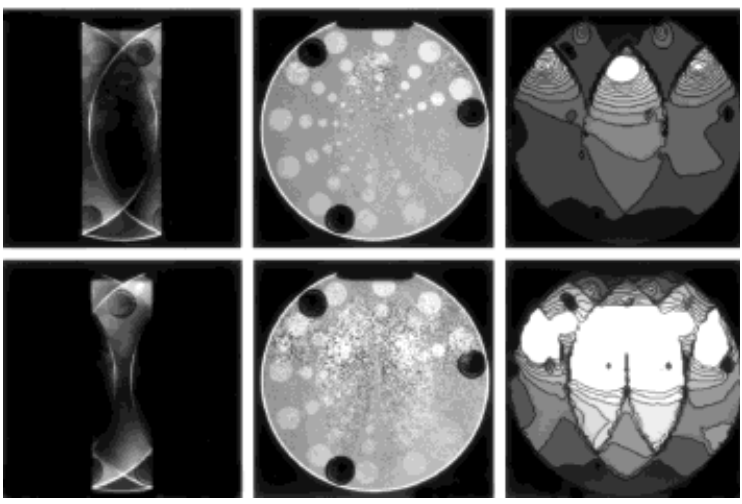

FIG. 5. SENSE imaging of a quality phantom with increasing reduction factor $R$ indicated on the left. Phase encoding in horizontal direction. Left: conventional sum-of-squares images. Middle: SENSE reconstruction from the same data. Right: maps of relative noise level as predicted by SENSE theory, using the same gray-scale as in Fig. 4.

References of the same FOV were acquired with an FFE sequence. The resulting SENSE images are displayed in Fig. 7 together with one of the intermediate single coil images. Except for the inevitable drawback in SNR, no loss of quality whatsoever is observed in the image acquired in $85 \mathrm{sec}$ instead of $170 \mathrm{sec}$.

For a cardiac application the five-coil array was positioned around a volunteer's chest in the fashion depicted in Fig. 3c. An electrocardiographically triggered echoplanar imaging (EPI) sequence (matrix $128 \times 128$, FOV 270 $\mathrm{mm}$, slice thickness $10 \mathrm{~mm}$, flip angle $30^{\circ}$, 6 echoes per excitation, half-Fourier acquisition) was used to acquire 24 heart phases in a double-oblique short-axis view. Reduction factors of $R=1.0$ and $R=2.9$ lead to breath-holds of $15 H B$ and $5 H B$, respectively. All heart phases were reconstructed using the same sensitivity maps. Systolic sample images are shown in Fig. 8. Again, loss in SNR is the only appreciable drawback in the image acquired in one-third of full scan time. In the liver region, noise is particularly enhanced due to unfavorable sensitivity relations.

\section{DISCUSSION}

SENSE imaging has been successfully performed in combination with FFE, TSE, and half-Fourier EPI. Generally, the concept should apply to any mode of Cartesian sampling of $k$-space. However, since coil phases are vital for reconstruction, care must be taken with phase fidelity in all applications, especially using EPI and half-Fourier techniques. SENSE reconstruction from non-Cartesian sampling patterns (16) is still inconvenient due to high computation complexity. However, numerical optimization and foreseeable increases in CPU power may render this option practical in the future. In addition to these general remarks, several issues deserve more detailed discussion.

\section{Signal-to-Noise Ratio}

In SENSE the SNR concern is considered in several ways. The propagation of noise from sensitivity maps into the final image is avoided by map smoothing. In weak reconstruction, including the practical Cartesian case, the image with pixel-wise maximum SNR is selected in the domain of images fulfilling the voxel shape criterion. Furthermore, the exclusion of empty voxels from reconstruction according to the references has proved to be an efficient means of enhancing regional SNR. At first glance, the beneficial effect of excluding voxels may seem surprising. It may be understood, though, by considering that excluding a voxel reduces the number of scalar conditions in [15] and thus increases the degrees of freedom available for SNR optimization.

The SNR penalty for reducing scan time by sensitivity encoding has been derived explicitly for the Cartesian case. Not surprisingly, it has been found that local SNR is essentially bound by the square root of acquisition time. This limitation is equivalent to that faced if scan time is reduced by increasing speed in $k$-space with enhanced gradient systems. However, the local SNR in a SENSE image generally falls short of this bound to a degree that

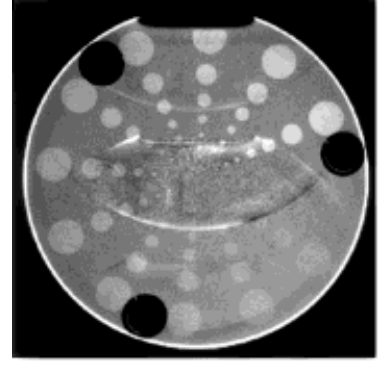

a

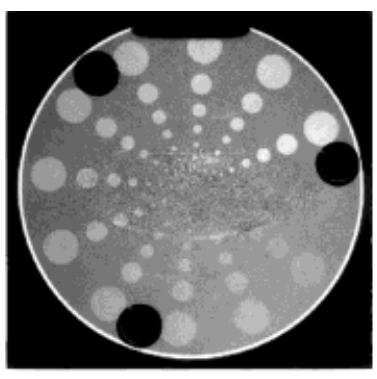

b

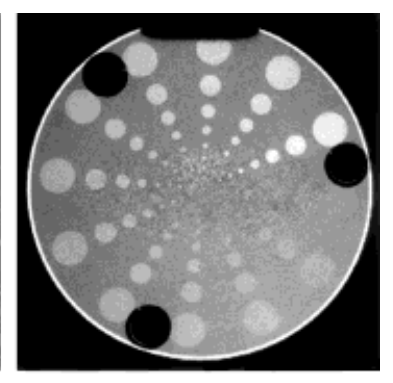

C
FIG. 6. The accuracy of sensitivity maps depends on the fitting order $P$. SENSE images based on sensivity maps obtained with (a) $P=0$, (b) $P=1$, and (c) $P=2$. 


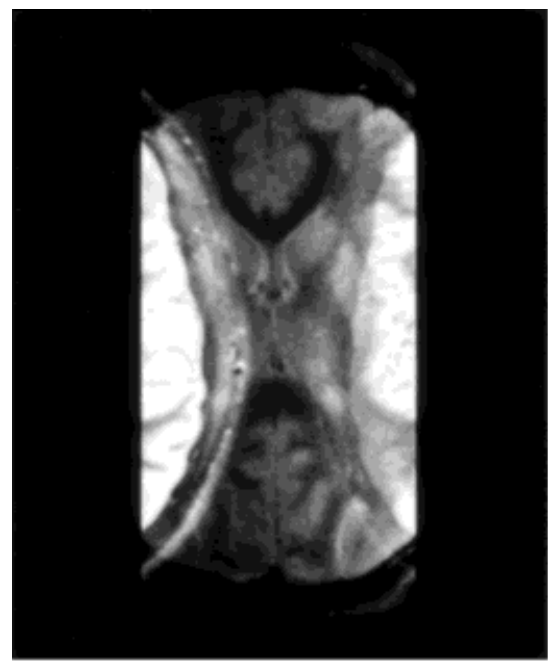

a

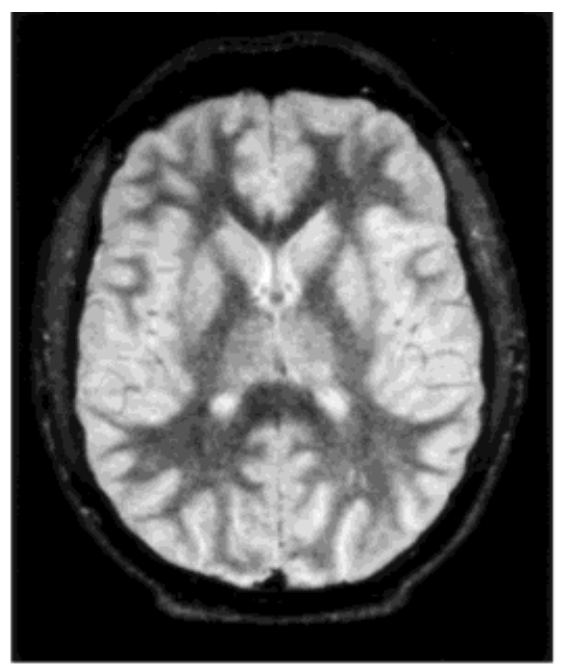

b

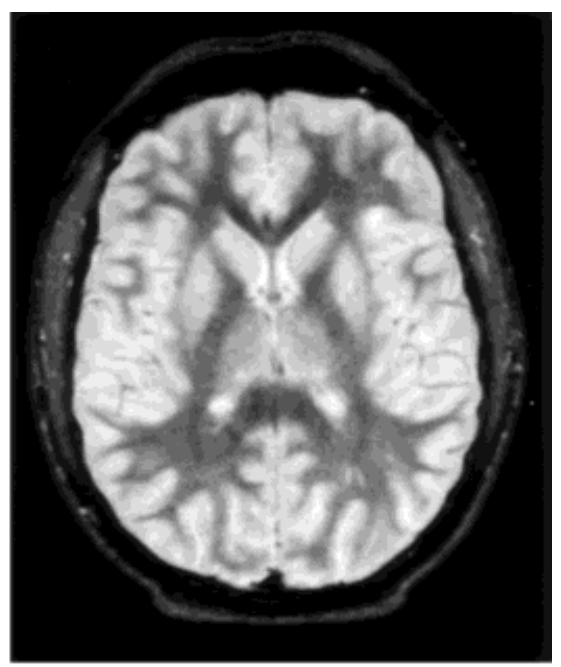

C

FIG. 7. Transverse brain images obtained with TSE using two coils. a, b: Reduction factor $R=2.0$, conventional single-coil image and SENSE reconstruction. c: SENSE image from fully Fourier encoded data.

depends on coil geometry. This effect inherent to sensitivity encoding is quantitatively described by the geometry factor introduced in this work. The geometry factor is a function of coil configuration and the reduction factor. In terms of SNR an optimal coil set-up is characterized by trade-offs among absolute sensitivity, single-channel noise levels, coil coupling, and geometrical sensitivity relations. Due to the specific role of coil geometry, dedicated sensitivity encoding arrays are called for, presenting coil making with a new design criterion.

\section{Sensitivity Maps}

For the determination of sensitivity maps, full-FOV reference images are required in addition to actual imaging. However, a sensitivity map obtained from one reference scan permits multiple SENSE imaging of the respective slice. In particular, map refinement includes short-range extrapolation; thus a single reference scan is sufficient even for handling multiple data sets of changing tissue configurations, as is encountered in cardiac imaging. The resolution of references in most cases may be lower than

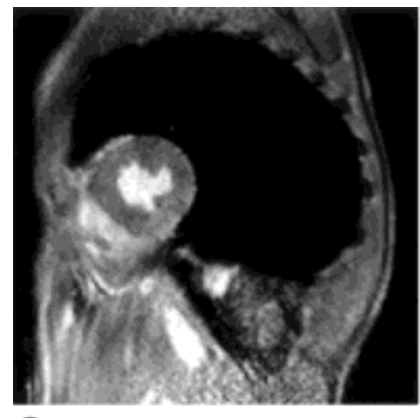

a

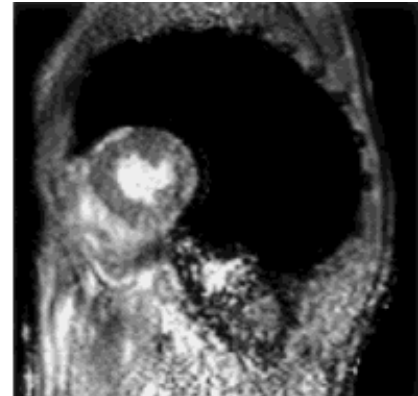

b
FIG. 8. Cardiac short-axis SENSE images acquired with half-Fourier EPI using five coils. The phase encoding direction is horizontal. a: Full Fourier encoding, 15 heartbeats. b: Reduction factor $R=2.9,5$ heartbeats. that of the final images. Full-resolution maps are then obtained by standard interpolation.

\section{Comparison With Previously Developed Techniques}

Among the methods summarized in the introduction the closest links to the SENSE concepts are found in the contribution by Ra et al. (6), which proposes a reconstruction algorithm exclusively for the case of Cartesian sampling. This technique is similar to the procedure derived in this work for the Cartesian case in that it combines DFT with an unfolding step. Major differences, however, consist in the application range of the reconstruction formulae, in the reference concepts, and in the fact that in Ref. 6 no means of SNR optimization are suggested. The technique described in Ref. 6 considers maximum reduction only, i.e., a reduction factor equal to the number of coils used. This requirement has been found to restrict application seriously, since geometry related noise enhancement in practical cases grows rapidly when the reduction factor approaches the maximum value (Figs. 4 and 5). As to sensitivity assessment, in Ref. 6 the use of images of a homogeneous phantom is suggested as estimates of coil sensitivity. This approach is hampered by the fact that coil sensitivity to a certain extent depends on load configuration.

A work published in 1993 by the same authors (17) suggests a related approach to image reconstruction, which, however, differs from both Ref. 6 and SENSE in that object contrast is kept in reference images, preventing sensitivity smoothing and extrapolation refinement.

The first successful experiments using parallel receivers for the purpose of scan time reduction were reported in 1997 by Sodickson et al., introducing the SMASH technique (7). SMASH uses Cartesian sampling with integer reduction and differs from the corresponding SENSE procedure in that it uses DFT in the last reconstruction step rather than in the first. The basic idea of SMASH reconstruction is to approximate full $k$-space data by linear combina- 
tion of multiple-coil sample values obtained in a reduced number of phase encoding steps. The weighting coefficients are determined by fitting linear combinations of coil sensitivities to harmonic functions of the desired FOV. In doing so it is crucial that low-order harmonics of the FOV can indeed well be approximated. Difficulty in fitting results in residual fold-over. Therefore, SMASH imaging requires particular coil arrays (18) and is restricted to suitable slice orientation (19). In SENSE the coil configuration can freely be optimized with respect to SNR, in particular with respect to the geometry factor. Note that geometry related noise enhancement is inherent to the coil arrangement and occurs in any linear reconstruction method, including SMASH.

As a consequence of the different degrees of freedom in choosing coil and slice geometry, the two methods differ in their reference strategies. SENSE requires full sensitivity maps, whereas in SMASH the reference demands are substantially less and can be fulfilled even by a little extra acquisition together with the aliased data (20).

\section{CONCLUSIONS}

Sensitivity encoding forms a valuable complement to gradient encoding. It permits considerable scan-time reduction with respect to standard Fourier imaging. The feasibility of SENSE imaging has been demonstrated in vitro and in vivo. In terms of practical value the most relevant characteristic of the technique is flexibility. Independently of coil and slice geometry, images free of specific artifacts are obtained. Nevertheless, the experimental set-up is a major determinant of SNR.

It has been shown that the SNR in SENSE images is governed by a fundamental limitation characterized by the square root of acquisition time. Hence the method is appropriate only when the need for scan speed outweighs SNR concerns. Therefore, rapid and real-time imaging is a promising application, as are all examinations that require breath-holding. In brain imaging, single-shot techniques are expected to benefit from shorter echo trains. Yielding high SNR at long scan times, 3D imaging with potentially 2D undersampling is another promising field of application.

At this stage it appears fair to say that sensitivity encoding is a promising concept in view of physiological and technical speed limits in $k$-space. However, the potential of the method to reduce scan time drastically in reasonable applications by factors of, say, 10 or more, remains to be investigated. According to SENSE theory, the major critical issue is SNR. Basic SNR can be enhanced to a certain degree by increasing the number of receiver coils. However, the joint effect of geometry factor and square-rootof-time penalty may ultimately prevent reduction factors much larger than those achieved in this work.

\section{ACKNOWLEDGMENTS}

The authors thank Dr. Peter Börnert and Dr. Tobias Schäffter from the Philips research laboratories in Hamburg, Germany, for aid in experiments and helpful discussions.
The valuable comments of Miha Fuderer from Philips Medical Systems in Best, The Netherlands, and of our student Roger Gösele are also gratefully acknowledged. Technical support was supplied by Philips Medical Systems in Best, The Netherlands, in particular by Hans Tuithof.

\section{APPENDIX A}

\section{Derivation of the Noise Matrices}

Assume that noise in acquisition channels arises from an arbitrarily large number of common, mutually independent noise sources $\xi_{\tau}(t)$, each with Gaussian statistics. Noise in the $\gamma$-th channel, $\eta_{\gamma}(t)$, is modeled as an individually weighted sum of the sources:

$$
\eta_{\gamma}(t)=\sum_{\tau} \omega_{\gamma, \tau} \xi_{\tau}(t)
$$

where $\omega_{\gamma, \tau}$ denotes time-independent, complex weighting coefficients. According to Eq. [6], the variance $\sigma_{\rho}^{2}$ of noise in the $\rho$-th voxel value is then given by

$$
\sigma_{\rho}^{2}=\sum_{\tau} \sigma_{\tau}^{2}\left|\sum_{\gamma, \kappa} F_{\rho,(\gamma, \kappa)} \omega_{\gamma, \tau}\right|^{2},
$$

where $\sigma_{\tau}$ denotes the standard deviation of the $\tau$-th noise source. Expanding the right square term and rearranging yields

$$
\sigma_{\rho}^{2}=\sum_{\gamma, \gamma^{\prime}, \kappa, \kappa^{\prime}} F_{\rho,\left(\gamma^{\prime}, \kappa^{\prime}\right)} \Psi_{\gamma, \gamma^{\prime}} F_{\rho,(\gamma, \kappa)}^{*}
$$

with the $n_{\mathrm{C}} \times n_{\mathrm{C}}$ receiver noise matrix $\Psi$ defined by

$$
\Psi_{\gamma, \gamma^{\prime}}=\sum_{\tau} \sigma_{\tau}^{2} \omega_{\gamma, \tau} \omega_{\gamma^{\prime}, \tau}^{*}
$$

Thus, $\sigma_{\rho}^{2}$ may be considered the $\rho$-th diagonal element of the image noise matrix $X$

$$
X=F \tilde{\Psi} F^{H}
$$

where

$$
\tilde{\Psi}=\Psi \otimes I d_{n_{K}}
$$

is referred to as the sample noise matrix. $\Psi$ can be determined experimentally in the following fashion. Consider the variance of the sum of two channels $\gamma, \gamma$ ' with a phase shift of $\varphi$ :

$$
\sigma^{2}\left(\eta_{\gamma}+e^{i \varphi} \eta_{\gamma^{\prime}}\right)=\sum_{\tau} \sigma_{\tau}^{2}\left|\omega_{\gamma, \tau}+e^{i \varphi} \omega_{\gamma^{\prime}, \tau}\right|^{2}
$$

Expanding the square term and rearranging using Eq. [A4] yields

$$
2 \operatorname{Re}\left(e^{i \varphi} \Psi_{\gamma, \gamma^{\prime}}\right)=\sigma^{2}\left(\eta_{\gamma}+e^{i \varphi} \eta_{\gamma^{\prime}}\right)-\sigma^{2}\left(\eta_{\gamma}\right)-\sigma^{2}\left(\eta_{\gamma^{\prime}}\right)
$$


With $\varphi=0$ for the real part and $\varphi=-\pi / 2$ for the imaginary part of $\Psi_{\gamma, \gamma}$ we obtain

$$
\begin{aligned}
\Psi_{\gamma, \gamma^{\prime \prime}}=\frac{1}{2}\left\{\sigma^{2}\left(\eta_{\gamma}+\eta_{\gamma^{\prime}}\right)+\right. & i \sigma^{2}\left(\eta_{\gamma}-i \eta_{\gamma^{\prime}}\right) \\
& \left.-(1+i)\left[\sigma^{2}\left(\eta_{\gamma}\right)+\sigma^{2}\left(\eta_{\gamma^{\prime}}\right)\right]\right\}
\end{aligned}
$$

Using this expression, the receiver noise matrix is readily determined from a reasonably large set of samples reflecting mere noise.

\section{APPENDIX B}

\section{Least-Squares Approximation of Ideal Voxel Functions}

Each voxel function must be a linear combination of encoding functions:

$$
f_{\rho}(\boldsymbol{r})=\sum_{\gamma, \kappa} F_{\rho,(\gamma, \kappa)} e_{\gamma, \kappa}(\boldsymbol{r}) .
$$

The best approximation of the ideal counterpart is characterized by

$$
\Delta_{\rho}=\int_{\mathrm{VOI}}\left|f_{\rho}(\boldsymbol{r})-i_{\rho}(\boldsymbol{r})\right|^{2} d \boldsymbol{r}=\min ,
$$

implying

$$
\frac{\partial \Delta_{\rho}}{\partial F_{\rho,(\gamma, \kappa)}}=0 \quad \forall \rho, \gamma, \kappa
$$

Calculating derivatives and rearranging terms yields

$$
\sum_{\gamma^{\prime}, \kappa^{\prime}}\left(F_{\rho,\left(\gamma^{\prime}, \kappa^{\prime}\right)} \int_{\mathrm{VOI}} e_{\gamma, \mathrm{\kappa}}^{*}(\boldsymbol{r}) e_{\gamma^{\prime}, \kappa^{\prime}}(\boldsymbol{r}) d \boldsymbol{r}\right)=\int_{\mathrm{VOI}} e_{\gamma, \mathrm{\kappa}}^{*}(\boldsymbol{r}) i_{\rho}(\boldsymbol{r}) d \boldsymbol{r}
$$

Using definition [11] this equation can be rewritten in matrix form:

$$
F C=E^{H},
$$

where

$$
C_{(\gamma, \kappa),\left(\gamma^{\prime}, \kappa^{\prime}\right)}=\int_{\mathrm{VOI}} e_{\gamma^{\prime}, \kappa^{\prime}}^{*}(\boldsymbol{r}) e_{\gamma, \kappa}(\boldsymbol{r}) d \boldsymbol{r}
$$

\section{APPENDIX C}

Minimization of Final Image Noise Under the Weak Voxel Condition

The minimum of $X_{\rho, \rho}$ under the weak voxel condition [15] is characterized by a corresponding absolute minimum of the Lagrange function

$$
L_{\rho}=X_{\rho, \rho}+\sum_{\rho^{\prime}} \Lambda_{\rho, \rho^{\prime}}(\mathrm{FE}-I d)_{\rho, \rho^{\prime}},
$$

where $\Lambda_{\rho, \rho^{\prime}}$ are Lagrange multiplicators. Using expression [A5] for $X$ and requiring that all partial derivatives of $L_{\rho}$ be equal to zero we obtain

$$
\begin{gathered}
2 \sum_{\gamma^{\prime}} F_{\rho,\left(\gamma^{\prime}, \kappa\right)} \Psi_{\gamma^{\prime}, \gamma}+\sum_{\rho^{\prime}} \Lambda_{\rho, \rho^{\prime}}^{*} E_{(\gamma, \kappa), \rho^{\prime}}=0 \quad \forall \rho, \gamma, \kappa \\
\sum_{\gamma, \kappa} F_{\rho,(\gamma, \kappa)} E_{(\gamma, \kappa), \rho^{\prime}}=\delta_{\rho, \rho^{\prime}} \quad \forall \rho, \rho^{\prime}
\end{gathered}
$$

In matrix form Eqs. [C2] and [C3] read

$$
\left(\begin{array}{cc}
2 \tilde{\Psi} & E \\
E^{H} & 0
\end{array}\right)\left(\begin{array}{l}
F^{H} \\
\Lambda^{H}
\end{array}\right)=\left(\begin{array}{c}
0 \\
I d
\end{array}\right)
$$

where $\tilde{\Psi}$ is defined according to Eq. [A6]. If $E$ and $\tilde{\Psi}$ have maximal rank the inverse of the square matrix on the left exists and is given by

$\left(\begin{array}{cc}2 \tilde{\Psi} & E \\ E^{H} & 0\end{array}\right)^{-1}$

$$
=\left(\begin{array}{cc}
\frac{1}{2} \tilde{\Psi}^{-1}\left(I d-E\left(E^{H} \tilde{\Psi}^{-1} E\right)^{-1} E^{H} \tilde{\Psi}^{-1}\right) & \tilde{\Psi}^{-1} E\left(E^{H} \tilde{\Psi}^{-1} E\right)^{-1} \\
\left(E^{H} \tilde{\Psi}^{-1} E\right)^{-1} E^{H} \tilde{\Psi}^{-1} & -2\left(E^{H} \tilde{\Psi}^{-1} E\right)^{-1}
\end{array}\right) .
$$

Right multiplication with Eq. [C4] yields

$$
F=\left(E^{H} \tilde{\Psi}^{-1} E\right)^{-1} E^{H} \tilde{\Psi}^{-1}
$$

\section{APPENDIX D}

\section{Derivation of DFT Based Reconstruction in the Cartesian Case}

Here the practical procedure described at the beginning of the Theory and Methods section is derived from the weak reconstruction formula [16], assuming Dirac distributions as ideal voxel functions. Consider sampling and voxel positions forming regular grids in 2D $k$-space and image domain, respectively. The grid constants shall be chosen such as to yield a resolution of $\Delta x, \Delta y$. Let the numbers of sampling positions and voxels be given independently by $n_{\mathrm{K}}=n_{\mathrm{Kx}} \times n_{\mathrm{Ky}}$ and $n_{\mathrm{V}}=n_{\mathrm{Vx}} \times n_{\mathrm{V} y}$, respectively. Then the grids are given by

$$
\begin{aligned}
& \boldsymbol{k}_{\kappa}=2 \pi\left(\frac{1}{\Delta x}\left(\frac{\kappa_{x}}{n_{K x}}-\frac{1}{2}\right), \frac{1}{\Delta y}\left(\frac{\kappa_{y}}{n_{K y}}-\frac{1}{2}\right)\right)^{T} \\
& \boldsymbol{r}_{\rho}=\left(\Delta x\left(\rho_{x}-\frac{n_{V x}}{2}\right), \Delta y\left(\rho_{y}-\frac{n_{V y}}{2}\right)\right)^{T},
\end{aligned}
$$

where 2D indices are used for convenience:

$$
\begin{aligned}
& \kappa=\left(\kappa_{x}, \kappa_{y}\right), \kappa_{x}=0, \ldots, n_{K x}-1, \kappa_{y}=0, \ldots, n_{K y}-1 \\
& \rho=\left(\rho_{x}, \rho_{y}\right), \rho_{x}=0, \ldots, n_{V x}-1, \rho_{y}=0, \ldots, n_{V y}-1 .
\end{aligned}
$$


Decompose $E$ as given by Eqs. [19] and [5] into the harmonic and the sensitivity component:

$$
E=\sqrt{n_{K}} \tilde{H}^{H} \tilde{S}
$$

where $\tilde{S}$ is a sparse, banded $\left(n_{\mathrm{C}} n_{\mathrm{K}}\right) \times n_{\mathrm{V}}$ matrix of sensitivity values:

$$
\begin{aligned}
\tilde{S}_{(\gamma, \lambda), \rho} & =s_{\gamma}\left(\boldsymbol{r}_{\rho}\right) \delta_{\lambda_{x}, \rho_{x} \bmod n_{K x}} \delta_{\lambda_{y}, \rho_{y} \bmod n_{K y}}, \\
\lambda & =\left(\lambda_{x}, \lambda_{y}\right), \lambda_{x}=0, \ldots, n_{K x}-1, \lambda_{y}=0, \ldots, n_{K y}-1
\end{aligned}
$$

and $\tilde{H}$ is a unitary block-diagonal matrix with one Fourier block for each coil,

$$
\tilde{H}=I d_{n_{C}} \otimes H
$$

Here the $n_{\mathrm{K}} \times n_{\mathrm{K}}$ Fourier matrix $H$ is defined by

$$
H_{\rho, \lambda}=\frac{e^{i k_{\lambda} r_{\rho}}}{\sqrt{n_{K}}},
$$

where, as opposed to Eqs. [D1] and [D2], the subscripts have the same ranges,

$$
\lambda_{x}, \rho_{x}=0, \ldots, n_{K x}-1 \quad \lambda_{y}, \rho_{y}=0, \ldots, n_{K y}-1 .
$$

Using the above decomposition and considering that $\tilde{\Psi}, \tilde{H}$ commute according to their definitions, the reconstruction matrix [16] is rewritten as

$$
F=\frac{1}{\sqrt{n_{K}}} \tilde{U} \tilde{H},
$$

where

$$
\tilde{U}=\left(\tilde{S}^{H} \tilde{\Psi}^{-1} \tilde{S}\right)^{-1} \tilde{S}^{H} \tilde{\Psi}^{-1}
$$

In Eq. [D10] $\tilde{H}$ is a matrix representation of coil-wise DFT, the first step in the practical procedure. The $2 \mathrm{D}$ index $\lambda$ of the matrix $\tilde{S}$ counts the voxels in the reduced FOV resulting from DFT. Due to its banded structure the matrix $\tilde{S}$ disintegrates into $n_{\mathrm{K}}$ partial matrices, which operate independently. The $\lambda$-th partial matrix comprises the $n_{\mathrm{C}}$ row indices $(\gamma, \lambda)$, with $\gamma$ varied, and the column indices $\rho=\left(\rho_{x}, \rho_{y}\right)$, which fulfil

$$
\rho_{x} \bmod n_{K x}=\lambda_{x} \quad \text { and } \quad \rho_{y} \bmod n_{K y}=\lambda_{y} .
$$

These column indices correspond to the voxels superimposed in the voxel $\lambda$ of the reduced FOV. According to its definition $\tilde{\Psi}$ obviously also acts independently upon each set of indices belonging to one $\lambda$. Thus, for every $\lambda$ formula [D11] can be evaluated separately, yielding the $\lambda$-th partial unfolding matrix

$$
U=\left(S^{H} \Psi^{-1} S\right)^{-1} S^{H} \Psi^{-1},
$$

where the transition to partial matrices is indicated by removing the tildes. The remaining scalar factor in Eq. [D10] is not important for image reconstruction but plays a role in noise considerations. Combining Eqs. [A5] and [D10], we find the partial image noise matrix for the $\lambda$-th unfolding step,

$$
X=\frac{1}{n_{K}}\left(S^{H} \Psi^{-1} S\right)^{-1}
$$

\section{REFERENCES}

1. Lauterbur PC. Image formation by induced local interactions: examples employing nuclear magnetic resonance. Nature 1973;242:190-191.

2. Roemer PB, Edelstein WA, Hayes CE, Souza SP, Mueller OM. The NMR phased array. Magn Reson Med 1990;16:192-225.

3. Hutchinson M, Raff U. Fast MRI data acquisition using multiple detectors. Magn Reson Med 1998;6:87-91.

4. Kwiat D, Einav S. A decoupled coil detector array for fast image acquisition in magnetic resonance imaging. Med Phys 1991;18:251265.

5. Kelton JR, Magin RL, Wright SM. An algorithm for rapid image acquisition using multiple receiver coils. In: Proceedings of the SMRM 8th Annual Meeting, Amsterdam, 1989. p 1172.

6. Ra JB, Rim CY. Fast imaging method using multiple receiver coils with subencoding data set. In: Proceedings of the SMRM 10th Annual Meeting, San Francisco, 1991. p 1240.

7. Sodickson DK, Manning WJ. Simultaneous acquisition of spatial harmonics (SMASH): ultra-fast imaging with radiofrequency coil arrays. Magn Reson Med 1997;38:591-603.

8. Pruessmann KP, Weiger M, Scheidegger MB, Boesiger P. Coil sensitivity encoding for fast MRI. In: Proceedings of the ISMRM 6th Annual Meeting, Sydney, 1998. p 579.

9. Weiger M, Pruessmann KP, Scheidegger MB, Boesiger P. Accelerated cardiac breathhold imaging using coil sensitivity encoding. In: Proceedings of the ISMRM 6th Annual Meeting, Sydney, 1998. p 799.

10. Weiger M, Scheidegger MB, Pruessmann KP, Boesiger P. Cardiac real-time acquisition using coil sensitivity encoding. In: Proceedings of the ISMRM 6th Annual Meeting, Sydney, 1998. p 803.

11. Brey WW, Narayana PA. Correction for intensity falloff in surface coil magnetic resonance imaging. Med Phys 1987;15:241-245.

12. Carlsen IC, Jensen D. Reconstruction algorithm for images obtained with flexible multi-element synergy coils. In: Proceedings of the SMR 2nd Annual Meeting, San Francisco, 1994. p 835.

13. Murakami JW, Hayes CE, Weinberger E. Intensity correction of phasedarray surface coil images. Magn Reson Imaging 1996;35:585-590.

14. Pruessmann KP, Weiger M, Scheidegger MB, Boesiger P. Coil sensitivity maps for sensitivity encoding and intensity correction. In: Proceedings of the ISMRM 6th Annual Meeting, Sydney, 1998. p 2087.

15. Haselgrove J, Prammer M. An algorithm for compensation of surfacecoil images for sensitivity of the surface coil. Magn Reson Imaging 1986;4:469-472.

16. Pruessmann KP, Weiger M, Boernert P, Boesiger P. Spiral SENSE: sensitivity encoding with arbitrary k-space trajectories. In: Proceedings of the ISMRM 7th Annual Meeting, Philadelphia, 1999. p 94.

17. Ra JB, Rim CY. Fast imaging using subencoding data sets from multiple detectors. Magn Reson Med 1993;30:142-145.

18. Griswold A, Jakob PM, Edelman RR, Sodickson DK. An RF array designed for cardiac SMASH imaging. In: Proceedings of the ISMRM 6th Annual Meeting, Sydney, 1998. p 437

19. Jakob PM, Griswold MA, Edelman RR, Manning WJ, Sodickson DK Cardiac imaging with SMASH. In: Proceedings of the ISMRM 6th Annual Meeting, Sydney, 1998. p 16.

20. Jakob PM, Griswold MA, Edelman RR, Sodickson DK. AUTO-SMASH, a self-calibrating technique for SMASH imaging. In: Proceedings of the ISMRM 6th Annual Meeting, Sydney, 1998. p 1975. 